\title{
Classification and Numbering on Posterior Dental Radiography using Support Vector Machine with Mesiodistal Neck Detection
}

\author{
AZ Arifin, M Hadi, A Yuniarti, \\ W Khotimah, AY Wijaya \\ Department of Informatics, Faculty of Inf Technology \\ Institut Teknologi Sepuluh Nopember (ITS) \\ Surabaya, Indonesia \\ Email: agusza@cs.its.ac.id
}

\author{
ER Astuti \\ Faculty of Dentistry \\ Airlangga University \\ Surabaya, Indonesia \\ Email: e_renwi_a@yahoo.com
}

\begin{abstract}
Dental radiography meets challenge to classify the dents into the proper class which useful for forensic and biomedical application. This paper proposed a novel method of classification and numbering on posterior dental radiography using support vector machine (SVM) with mesiodistal neck detection. In this method we developed SVM using a nouvelle feature with mesiodistal neck teeth. This feature was used to solve the problem in the dental image which suffered with completeness of whole part of teeth (crown - root). Preprocessing for enhancements included morphological operation, contrast adaptive, and tresholding.. Every tooth has been assigned according to universal dental numbering and classified as their sequence order. Our system achieved classification precision of $90 \%$. This approach is robust and optimal for solving the problem of dental classification.
\end{abstract}

Keywords- Support Vector Machine, Mesiodistal, dental, Image processing, Morphological filtering, Teeth Numberin

\section{INTRODUCTION}

In the forensic domain, dents are one of important aspects to analyze victims. Dents have a special characteristic which can be used to make sure the identification of victims. Although, identifying using dents is secure-less but in several cases, for example, in tsunami, natural disaster or even traffic accident, which give serious defect to the victim's body, so it Is too difficult to analyze using another part of body. In those cases, human body usually gets serious damage, except some strong parts, and one of them is teeth. Therefore, teeth are chosen as the identification factor to help the forensic investigation[2].

Every tooth has its own information; the information must be extracted for the identification purposes. The information is used for classification. This step distinguishes dental into their type and number.

Some researchers have been done to solve the problem of classification. Mahammad had presented an approach to classify and give number to the teeth bitewing image. They used Bayesian classification to distinguish molars and premolars teeth, and number as the international standard numbering teeth [1]. Fourier descriptor of dental bitewing image was used as the feature of the classification model.
This approach was success until it met the limits in several cases. It failed to classify some teeth which have no root in their image. Using the Fourier descriptor of contour also has weakness, such as when the crown condition of both molar and premolar is identical, It is difficult to distinguish them by using this feature. This error has a big influence in the final step, numbering. Finally it decreases the total accuracy of classification system.

In order to overcome the previous problem, Lin and Lay proposed teeth region and contour information for dental classification [2]. This algorithm provided a binary linear support vector machine (SVM) instead of Bayesian. And it used length-width ratio as the feature. This algorithm perform well except for some condition that we cannot rely on the length-width ratio of several images, due to the broken teeth, uncompleted image, i.e. without root, or even the problem of differentiation of root and gums.

In other research, Faraein used mesiodistal neck as feature of the current classification model [1]. This method had stability in age raising and teeth broken. However the robustness in various type of dental image was not considered.

In this paper, an improved approach was presented to overcome the previous problem of teeth classification, in various type of dental image. We used bitewing and panoramic image to ensure the robustness of this method in larger space. A complete segmentation method and SVM approach with mesiodistal neck of bitewing and panoramic as feature were proposed to build a robust teeth classification system.

The detail of this approach will be provided in the next section. We will explain the proposed method; the brief explication of each step, then the experimental result and data analysis, and the last was conclusion.

\section{PROPOSED METHOD}

The idea of our proposed method was shown in Fig. 1. The input of this system was dental image. Top and bottom hat, iterative thresholding, integral projection, and 8-connected component were used for preprocessing. Neck detection was applied for feature extraction. And the last steps were 


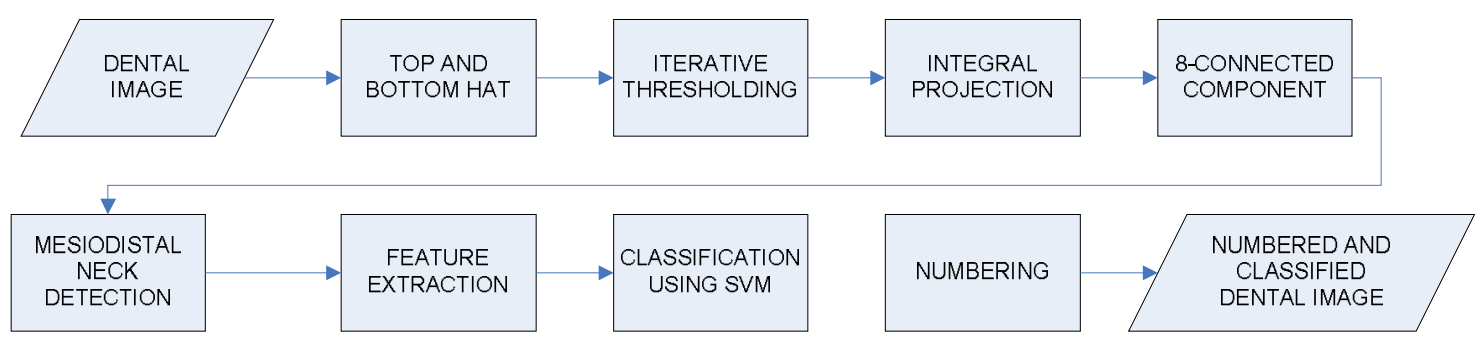

Figure 1 The Block Diagram of proposed method

\section{A. Dental Image Segmentation}

There are three main phases in this step that should be done to obtain a proper feature for the classification process. Those phases are image enhancement, image binarization, and teeth separation.

There are three main phases in this step that should be done to obtain a proper feature for the classification process. Those phases are image enhancement, image binarization, and teeth separation.

Image enhancement is one of the most important steps because the accuracy of the classification is depending on the quality of the image. It make the quality of image is as proper as possible to obtain the expected feature. In this research, some enhancement methods were utilized, such as top-hat bottom-hat morphology and Contrast-limited adaptive histogram. They were applied to achieve a distributed contrast and reduce noise.

Image binarization is a process of transforming grey level image into binary image. This process is used to separate the teeth region (foreground), image background, gums, and undesirable region that can provoke noise.

An adaptive tresholding was used to change gray scale image into black and white image. In addition, some morphological action has been done, e.g. closing and opening to minimize the number of noises during this phase.

The objective of teeth separation is to find ROI of the tooth. This process separated the maxilla and mandibular using horizontal projection. While each tooth was separated using vertical projection. Then 8 connected components was used to create small region that consist only each single tooth.

\section{B. Dental Image Classification}

The main objective of this paper is designing classification model using Support vector machine (SVM) for assigning teeth in which class they should be and what number they have. This current research proposes a feature called Mesiodistal Neck to support the classification.

Before explaining the method how to classify, it is necessaries to understand the contribution of Mesiodistal neck teeth. A Mesiodistal Neck tooth, as shown in Fig. 2, is a line between cementum and enamel, which separates the crown and root of tooth. Mesiodistal neck seems more stable again aging and gum disease. Several studies use only crown, whereas we have known that some teeth has similar form of crown. In that case, crown can be the only variable to depend. As well the root tooth, in some cases we cannot figure the root because it is blur and has unclear boundary with the gum.

\section{Proposed Mesiodistal Neck Teeth Detection}

This tooth neck is a place where the crown has tight width and the root has wider width. In normal tooth, the width is stable, around $1 / 3$ from the base of the root.

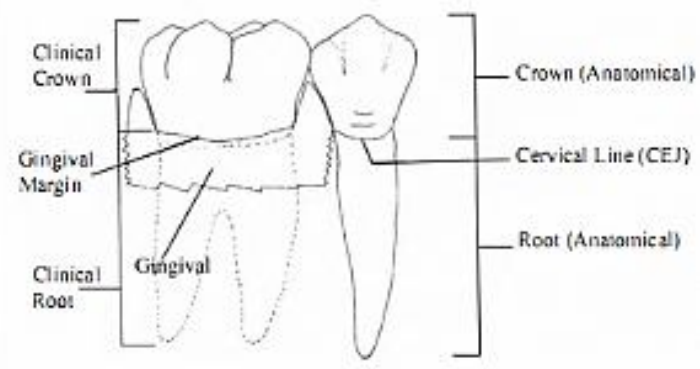

Figure 2 The Description of Mesiodistal Neck [3]

Normal teeth usually show an image which stand for 90 , so we can detect easily the spot of the neck. But in many cases, teeth are not shown vertically. They create an angle with the xaxis. In order to achieve the best extraction of the teeth neck, a rotation is required before the width counting.

First we have to count the skew angle of the tooth $(\alpha)$, and then represent this angle into a degree between $-90^{\circ}$ and $90^{\circ}$. In order to make vertical tooth, we can rotate the object into -90 。 or $90^{\circ}$. If $\alpha<0$, we rotate the object into $-90^{\circ}$ as the closest point to make the object vertical. Otherwise, if $\alpha>0$, we rotate the object with $90^{\circ}$. Then we can count the width of the tooth precisely.

After having the rotated teeth, the width of each tooth can be counted as the formula 1 and a group of width can also be promoted as the mesiodistal.

$$
\text { Mesio Neck }[i]=\operatorname{Max}\left(y_{i}\right)-\operatorname{Min}\left(y_{l}\right)
$$

where $\mathrm{i}$ is the order of row in the tooth contour, maximum and minimum column in the row i-th, shown by $\operatorname{Max}(y i)$ and Min (yi). 
The group of those widths in Mesiodistal Neck can be represented in a histogram. The sample of the histogram is shown in Fig. 3. The characteristic of Mesiodistal neck stated in the previous section is used to find Neck location. The histogram, in Figure 3, shows some points that can be stated as extreme point. The mesiodistal neck is found when the previous point has a decreasing pattern of width and it shows a stable pattern. It is the narrowest point between the crown and root. Then the found width is used as mesiodistal neck value and its position is saved as the neck line of the tooth. After recording the value of mesiodistal neck of each tooth, the classification is processed.

\section{Proposed Classification Method}

In this paper, supervised learning is used to classify the teeth into molar or premolar. SVM or Support Vector Machine was used because it is robust, and simple. The basic idea of SVM is to transform data into higher dimensional space and to find an optimal hyper plane with maximal separation margin between classes.

In SVM, data is assumed linearly separable. The hyperplane of our binary SVM as the general form, is shown as $\mathrm{w}^{\circ} \mathrm{x}+\mathrm{b}=0$, where $\mathrm{w}$ is the normal to the hyperplane and $\mathrm{b} /\|\mathrm{w}\|$ is the perpendicular distance from the hyperplane to the origin.

A linear SVM works as follows. First, $\mathrm{N}$ data, $(x i, y i)(i=1, \ldots \ldots, N)$, are randomly selected from the whole data set as the training set for finding the optimal $\mathrm{w}^{*}$ and $\mathrm{b}^{*}$ such that the hyperplane $\mathrm{w}^{*}{ }^{\circ} \mathrm{x}+\mathrm{b}^{*}=0$ is as far as possible from the closest members of both classes. The training data $(\mathrm{x}, \mathrm{y})$, support vectors, will fall on either side of the two planes B 1 : w ${ }^{\circ} \mathrm{x}+\mathrm{b}=1$ and $\mathrm{B} 2: \mathrm{w}{ }^{\circ} \mathrm{x}+\mathrm{b}=-1$. The Hyperplane $\mathrm{w}{ }^{\circ} \mathrm{x}+\mathrm{b}=0$ is decision boundary of this binary linear classifier.

Each training data is stated by a tuple $(x i, y i)(i=1, \ldots \ldots, N)$, where $\mathrm{xi}=(\mathrm{xi} 1, \mathrm{xi} 2, \ldots \ldots, \mathrm{xid}) \mathrm{T}$ corresponds to the feature vector (teeth Area L/W, relative Mesiodistal width)T, and yi $\varepsilon\{1$, $1\}$ denotes the class label, i.e., molar or premolar. This binary linear SVM model provoke the optimal $\mathrm{w}^{*}$ and $\mathrm{b}^{*}$ which can be calculated as formula 2 and 3 .

$W^{*}=\sum_{i=1}^{n} \alpha_{i} y_{i} x_{i}$

$b^{*}=\frac{1}{N} \sum_{S \in S}\left(y_{s}-\sum_{m \in S}^{n} \alpha_{m} y_{m} x_{\mathrm{ra}_{\mathrm{u}}}, x_{s}\right)$,

where $\alpha$ is the Lagrange multiplier such that is maximized, subject to the constraints and; $\mathrm{B}$ is a matrix with $\mathrm{Bij}=$ yiyjxi.xj, and $\mathrm{S}$ is the set of support vectors whose .

For testing, $\mathrm{y}^{\prime}=\operatorname{sgn}\left(\mathrm{w}^{*}{ }^{\circ} \mathrm{x}^{\prime}+\mathrm{b}^{*}\right)$ is the vector function where sgn is the sign function, $\mathrm{x}^{\prime}$ is the data test. Each data will belong to molar if $y^{\prime}>0$, otherwise it will be premolar.

\section{E. Teeth Numbering}

Teeth Numbering assign the classified teeth, Molar or Premolar, into a unique number according to universal teeth numbering system (started by 1 into 32)[4]. The classification results are formatted in a pattern of "P" or " $\mathrm{M}$ " that indicate the pattern of the current image. As the ground truth we have save the default pattern of posterior teeth both maxilla and mandibular. The input pattern for this phase should follow the sequence of the default format. Making a sequence format matching helps us assigning the number of each tooth. In the end of this step, we have the fixed number of each tooth according the universal teeth numbering system.

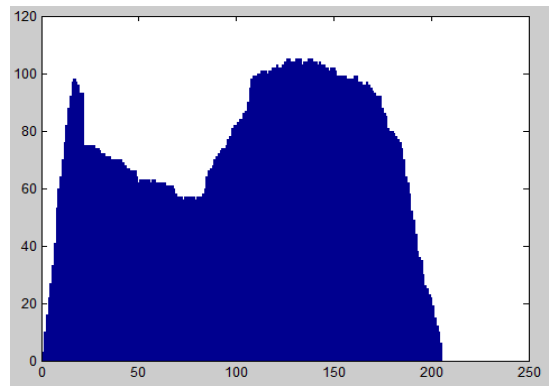

Figure 3 Histogram Representation of Mesiodistal Neck Width

\section{EXPERIMENT}

This proposed method was implemented in Matlab and LibSvm. This experiment gived three main results, the segmentation, classification, and Teeth Numbering. We used 15 dental images for training and testing. They consist of 110 single teeth with 60 and 50 of Molar and 50 Premolar respectively.

\section{A. Segmentation}

We can conclude that the algorithm of segmentation stand in its power because it shows a good accuracy of segmenting the teeth, as Fig. 4. The system gives a result, 95\% of total teeth can be recognized as teeth. In the failure case, the teeth give some blur gray scale, so it's difficult to distinguish between teeth and gums.

After having the ROI, we have to extract the Mesiodistal neck teeth, the example of result of the Mesiodistal are shown in Fig. 5. The images presented are proof that the approach has found the mesiodistal position in each tooth.

\section{B. Classification}

Classification as the main phase of this system also shows a good result. System can classify correctly 99 single teeth from 110 total teeth. 54 Molar teeth can be classified properly from 60 and 45 premolars from total 50 premolars. The detail is shown in Table 1. Some cases are found during the classification process. Images with the failure of the previous step are absolutely failed for this step, and it can be burden for the accuracy.

Moreover, several images can be distinguished properly. Some results are shown in Fig. 6. Because the size of the teeth is so similar, it's so possible for those teeth; they are placed in the marginal area of the Hyperplane, and may be they can be misclassified. 


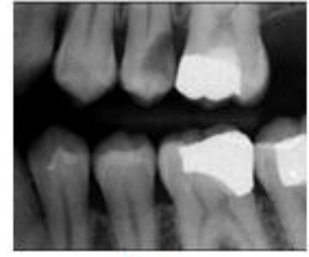

(a)

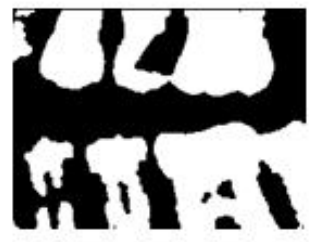

(c)

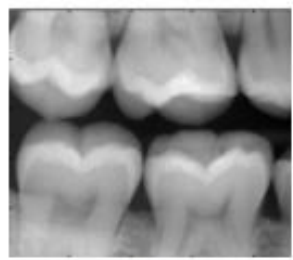

(b)

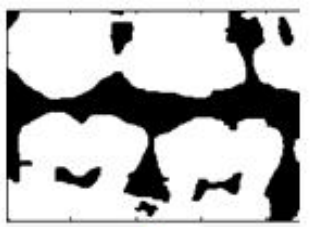

(d)
Figure 4 (a) and (c) the example of input and output of the un-segmented image, (b) and (d) is segmented perfectly

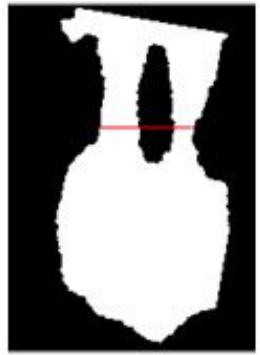

(a)

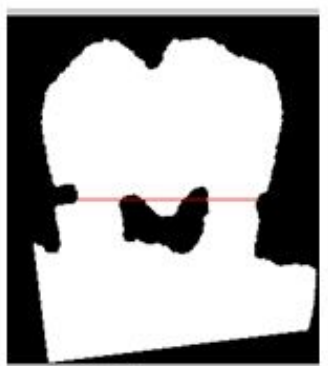

(b)
Figure 5 a) Mesiodistal for Maxilla tooth, 4b) for the mandibular

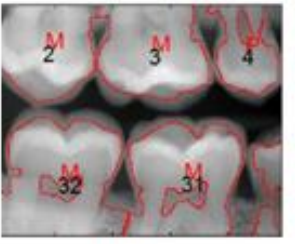

(a)

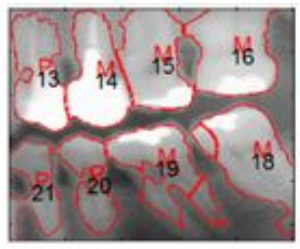

(c)

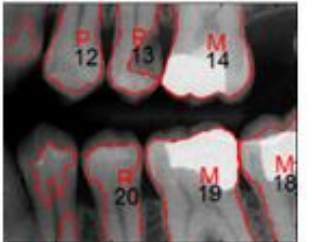

(b)

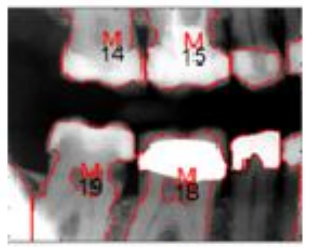

(d)
Figure 6 a) Normal Classified. b) Fail to segment some teeth, c) Tooth 14 should premolar (misclassified) d) fail to segment and there are ambiguous patterns $(\mathrm{MM})$
TABLE 1. ACCURACY OF THE CLASSIFICATION PROCESS

\begin{tabular}{|l|l|c|}
\hline \multirow{2}{*}{ Teeth } & \multicolumn{2}{|c|}{ Method } \\
\cline { 2 - 3 } & \multicolumn{2}{|c|}{ Pre } \\
\hline Molar & $87 \%$ & $90 \%$ \\
\hline Premolar & $88 \%$ & $90 \%$ \\
\hline Total & $87.5 \%$ & $90 \%$ \\
\hline
\end{tabular}

\section{Teeth Numbering}

The algorithm used in teeth numbering has an impact in classification result. The same data is analyzed and it shows the accuracy of numbering itself is around $86 \%$. It's caused by the pattern found in classification step is too ambiguous. For example, the Pattern MM is too difficult to decide whether it's the part of PPPMMM, PPPMMM or even the otherwise position. Although it has some lack in numbering but this approach can help the classification. When some bizarre patterns appear, such as PMPMM, according to the algorithm, this pattern will be repaired in their proper sequence order, PPPMMM. Therefore, the approach of numbering can increase the accuracy of classification up to $3 \%$ shown in Table 1 .

\section{CONCLUSION}

Teeth segmentation, classification and also numbering are still a main art of the dental radiograph or may be forensic research. Automatic identification of disaster victims is absolutely needed, respectively the rapid performance and high accuracy. In this paper, we propose an approach of classification using the mesiodistal line of teeth. The enhancement of dental image using the homomorphic filtering and contrast enhancement are given in the first part of methodology. This enhancement is followed by the adaptive threshold for transforming the gray image into binary. The Integral projection is chosen to separate the teeth and take the ROI. Our main approach in selecting the path of the mesiodistal is also shown in the previous section.

Our algorithm of classification applied has given high accuracy in various cases. It can overcome the most noise problem, distinguish the image into Premolar and Molar (90\% accuracy) and most teeth are assigned their number precisely. Blur Image with some weak gray level and some ambiguous patterns still need to be explored due to enhance the performance of the system. Some unexpected cases which have not been found are still big challenge to solve.

\section{REFERENCES}

[1]. Aeini, F., \& Mahmou, F. (2010). Classifcation and numbering of posterior teeth in bitewing dental images. 3rd International Conference on Advanced Computer Theory and Engineering(CTE).

[2]. Lin, P., Lai, Y., \& Huang, P. (2010). An Effective Classification and Numbering System for Dental Bitewing Radiographs using Teeth Region and Contour Information. Pattern Recognition Journal, Vol.43 , 13801392 . 
[3]. Mohammad, M., \& Mohamed, A.-M. (2005). Classifcation and numbering of teeth in dental bitewing images. Elsevier , $38: 577$ - 586.
[4]. Yuniarti, A., Nugroho, A., Amaliah, B., \& Arifin, A. (March 2012). Classification and Numberingof Dental Radiographs for an Automated Human Identification System. Telkomnika, Vol.10, No,1 , 137-146. 